\title{
MEAN PLATELET VOLUME PREDICTS INCREASED BLOOD TRANSFUSION REQUIREMENTS IN ADULT PATIENTS UNDERGOING LIVER TRANSPLANTATION
}

\author{
Starczewska MH1, Giercuszkiewicz D1, Piwowarska J1, Niewinski G1, \\ Krawczyk $\mathbf{M}^{2}$, Kanski $\mathbf{A}^{1}$
}

\author{
${ }^{1}$ Department of Anaesthesiology and Intensive Care Medicine, Medical University of Warsaw, Poland \\ 2 Department of General, Transplant and Liver Surgery, Medical University of Warsaw, Poland
}

Background: Despite advances in surgical technique and anaesthetic management, perioperative bleeding remains one of the main causes of increased mortality and morbidity in adult patients undergoing liver transplantation (LT). Few studies have suggested that greater mean platelet volume (MPV) can predispose to thrombotic events. However so far there were no studies to evaluate the predictive value of MPV on perioperative blood loss in pts undergoing solid organ transplantation.

Objectives: We sought to determine the prognostic value of MPV for blood transfusion requirements in adult patients undergoing LT.

Material and methods: We have prospectively enrolled 78 adult patients undergoing cadaveric LT at Medical University of Warsaw. Patients were recruited from August 2012 until March 2014. Written informed consent was obtained from all patients. Blood samples for MPV measurement were obtained at admission before LT. The primary end point was the number of units of red blood cells (RBC), fresh frozen plasma (FFP), platelet concentrates (PLT) and cryoprecipitate transfused during LT.

Results: Mean age in the study group was 49,1 $\pm 12,4$ years and 55,1\% were male patients. The most common aetiology of cirrhosis was viral hepatitis (47,4\%). Mean MELD score was 11,9 \pm 5,2 points.

Median duration of surgery was 410 min (260 - 690). In 6,4\% of cases a veno-venous bypass was employed. Median time of cold ischemia was 549 min (310 - 948). The median volume of transfused crystalloids and colloids intraoperatively was $3500 \mathrm{ml}(2500-7500)$ and $1500 \mathrm{ml}$ $(0-3500)$ respectively. Intraoperative transfusion of blood products in the study group is displayed in Table 1 .

Table 1. Intraoperative blood products transfusion.

\begin{tabular}{|l|c|c|c|}
\hline & Frequency & Median & Range \\
\hline Red blood cells & $79,5 \%$ & 3,5 & $0-25$ \\
\hline Fresh frozen plasma & $88,5 \%$ & 5 & $0-22$ \\
\hline Platelet concentrate & $19,2 \%$ & 0 & $0-2$ \\
\hline Cryoprecipitate & $2,6 \%$ & 0 & $0-8$ \\
\hline
\end{tabular}

The median value of MPV in the study group was 10,9 $\mathrm{fl}$ (range 7,2-13,1 fl). There was no correlation between MPV and platelet count.
Multivariate linear regression analysis showed that MPV was independently associated with the number of RBC transfused intraoperatively (Table 2).

Table 2. Multivariate linear regression analysis for intraoperative transfusion of RBC.

\begin{tabular}{|c|c|c|c|c|}
\hline & B & $\boldsymbol{\beta}$ & $95 \%$ CI & p \\
\hline Constant & 38,311 & & $24,971-51,651$ & 0,000 \\
\hline Haemoglobin (g/dl) & $-1,056$ & $-0,417$ & $-1,655--0,457$ & 0,001 \\
\hline MPV (fl) & $-1,069$ & $-0,234$ & $-2,106--0,32$ & 0,044 \\
\hline BMI $\left(\mathrm{kg} / \mathrm{m}^{2}\right)$ & $-0,268$ & $-0,226$ & $-0,546-0,009$ & 0,058 \\
\hline Platelet count $\left(* 10^{6} / \mu \mathrm{l}\right)$ & $-0,016$ & $-0,211$ & $-0,033-0,001$ & 0,064 \\
\hline
\end{tabular}

Similar analysis for intraoperative FFP transfusion showed a trend towards increased FFP requirements with decreased MPV $(\beta=-0,185$; $\mathrm{p}=0$,086). MPV did not predict intraoperative PLT transfusion requirements, however it was a significant predictor for cryoprecipitate transfusion during LT (Table 3).

Table 3. Multivariate linear regression analysis for intraoperative transfusion of cryoprecipitate.

\begin{tabular}{|l|c|c|c:c|}
\hline & $\boldsymbol{\beta}$ & $\boldsymbol{\beta}$ & $\mathbf{9 5 \%} \mathbf{C I}$ & $\mathbf{p}$ \\
\hline Constant & $-0,152$ & & $-3,218-2,914$ & 0,921 \\
\hline Bilirubin (mg/dl) & 0,188 & 0,416 & $0,090-0,286$ & $<0,001$ \\
\hline Duration of surgery (min) & 0,003 & 0,247 & $0,000-0,006$ & 0,024 \\
\hdashline Age (years) & 0,024 & 0,217 & $0,001-0,047$ & 0,045 \\
\hline MPV (fl) & $-0,251$ & $-0,236$ & $-0,482--0,021$ & 0,033 \\
\hline
\end{tabular}

Conclusions: This is the first study to demonstrate that mean platelet volume is significant and independent predictor of increased blood transfusion requirements during LT in adult patients. Except for its prognostic value it may also carry further therapeutic implications. However the mechanistic link of this observation needs to be further elucidated. 